\title{
Performance Evaluation of Wavelength Conversion Using a Wideband Semiconductor Optical Amplifier at $40 \mathrm{Gbit} / \mathrm{s}$
}

\author{
Y. Said", H. Rezig and A. Bouallegue
}

SYS' COM Research Laboratory, TIC Department, National Engineering School of Tunis (ENIT), Tunisia

\begin{abstract}
In this paper, we will report and characterize the impact of the nonlinear polarization rotation on the behavior of a wavelength converter based on cross-gain modulation effect in a semiconductor optical amplifier (SOA) at $40 \mathrm{Gbit} / \mathrm{s}$. Nevertheless, we investigate and evaluate its performance as function of the intrinsic and extrinsic SOA parameters, such as the bias current, the signal format, the input signal power and its polarization state that determine the magnitude of the polarization rotation by measuring the ellipticity and the azimuth. Also, the impact of noise effects on the structure behavior will be investigated through determining the noise figure. Particularly, we have focused on the performance of an improved wavelength conversion system via the analysis of quality factor and bit error rate referring to numerical simulation.
\end{abstract}

\section{INTRODUCTION}

All-optical wavelength converters are considered key components in future wavelength division multiplexing (WDM) optical networks due to their main advantage that consists of increasing the flexibility and the capacity of these networks and facilitating WDM network management. Either, they form an essential part of the wavelength routing switch that is employed in the all-optical buffering concept. All-optical wavelength conversion can be realized by using fiber nonlinearities or nonlinearities in semiconductor devices.

In the last few years, a considerable attention has been focusing on semiconductor optical amplifiers (SOAs) and their potential used in the optical communication systems. Especially, SOAs have generated more and more interest when optical signal processing is involved. Subsequently, they are exploited to achieve functional devices in wavelength conversion at high bit rates, which is a very important function in conjunction with WDM systems. This reason makes them also very useful in wavelength routers, which manage wavelength paths through optical networks based on complex meshes, rather than point-to-point architectures [1].

Many studies have paid more attention on SOAs performance for implementing and configuring wavelength conversion schemes. Hence, several optical wavelength converters based on SOA nonlinearities have been proposed and discussed, such as cross-gain modulation (XGM) [2,3], cross-phase modulation (XPM) [4,5], four-wave mixing (FWM) [6,7], and cross-polarization modulation (XPolM) $[1,8]$. Each configuration has its advantages and disadvantages; and then its framework of application in optical communication networks.

Optical wavelength converters based on SOA nonlinearities, which are fundamental components in today's photonic

*Address correspondence to this author at the SYS'COM Laboratory, National Engineering School of Tunis (ENIT), B.P. 37 Le Belvedere 1002 Tunis, Tunisia; Fax: +216 71872 729; Tel: +216 71875 475; E-mail 1: youssef_tn@yahoo.com; Email 2: Youssef.Said@ @unisietelecom.tn networks, offer some advantages in terms of integration potential, power consumption, and optical power efficiency. However, the major limitation of SOA-based wavelength converters is the slow SOA recovery, causing unwanted pattern effects in the converted signal, and limiting the maximum operation speed of the wavelength converters. It has already been theoretically and experimentally clarified that the increase in electrical pumping power, confinement factor, and interaction device length effectively improve the speed performance [9-10]. For improving the SOA-based wavelength converters, some techniques are proposed, such as: Fiber Bragg grating at $100 \mathrm{Gbit} / \mathrm{s}$ [3], interferometric configuration at $168 \mathrm{Gbit} / \mathrm{s}$ [11], two cascaded SOAs at $170.4 \mathrm{Gbit} / \mathrm{s}$ [12], and optical filtering at $320 \mathrm{Gbit} / \mathrm{s}$ [13].

XPolM effect in a SOA structure, which has been subject of many investigations in last years, is directly related to the transverse electric and transverse magnetic (TE/TM) mode discrepancy of XPM and XGM. In previous studies [14-15] treating this nonlinear effect, we have demonstrated and verified that SOA bias current, SOA length, input signal power and its polarization state are the parameters that determine the magnitude of the polarization rotation. We proceed in this paper to evaluate the influence of these parameters and the signal format (non return-to-zero "NRZ", return-to-zero "RZ") on the behavior of the SOA structure used in wavelength conversion configuration and to analyze the performance dependence on several critical operation parameters of the structure.

\section{CONCEPT OF WAVELENGTH CONVERSION BASED ON NONLINEAR EFFECTS IN A SOA}

All-optical wavelength conversion refers to the operation that consists of the transfer of the information carried in one wavelength channel to another wavelength channel in optical domain. It is a key requirement for optical networks, because it has basically to be used to extend the degree of freedom to the wavelength domain. Moreover, All-optical wavelength conversion is also indispensable in future optical packet switching (OPS) networks to optimize the network perfor- 
mance metrics, such as packet loss rate, packet delay, etc [16]. Also, it is very useful in the implementation of switches in WDM networks. In addition, it is crucial to lower the access blocking probability and therefore increasing the utilization efficiency of the network resources in wavelength routed optical networks.

While a significant part of network design, routing and wavelength assignment depends on the availability and performance of wavelength converters; and as many techniques have been explored and discussed in this context, alloptical wavelength converters based on SOA structure have attracted a lot of interest thanks to their attractive features, such as the small size, the fast carrier dynamics, the multifunctional aspect and the high potential of integration. The main features of a wavelength converter include its transparency to bit rate and signal format, operation at moderate optical power levels, low electrical power consumption, small frequency chirp, cascadability of multiple stages of converters, and signal reshaping.

When a RZ pump (the data signal) at wavelength $\lambda_{1}$ and a continuous wave $(\mathrm{CW})$ probe signal at wavelength $\lambda_{2}$ are injected into an SOA, the pump modulates the carrier density in its active region and hence its gain and refractive index. This leads to a change in the amplitude and phase of the CW probe signal. In the case of XGM, the output probe signal from the SOA carries the inverted modulation of the RZ input data signal.

To obtain an output probe signal with non-inverted modulation, XPM is used, whereby the phase modulation of the probe signal is converted to amplitude modulation by an interferometer. Particularly, in the wavelength conversion based on XGM scheme, a strong input signal is needed to saturate the SOA gain and thereby to modulate the $\mathrm{CW}$ signal carrying the new wavelength. While XGM effect is accompanied by large chirp and low extinction ratio, and limited by the relatively slow carrier recovery time within the SOA structure, impressive wavelength conversion of up to $40 \mathrm{Gbit} / \mathrm{s}$ and with some degradation even up to $100 \mathrm{Gbit} / \mathrm{s}$ [3], has been demonstrated.

To overcome the XGM disadvantages, SOAs have been integrated in interferometric configurations, where the intensity modulation of the input signal is transferred into a phase modulation of the CW signal and exploited for switching. These XPM schemes enable wavelength conversion with lower signal powers, reduced chirp, enhanced extinction ratios and ultra fast switching transients that are not limited by the carrier recovery time. Subsequently, wavelength conversion based on XPM effect with excellent signal quality up to $100 \mathrm{Gbit} / \mathrm{s}$, has been demonstrated [17] by using a fully integrated and packaged SOA delayed interference configuration that comprises a monolithically integrated delay loop, phase shifter and tunable coupler.

FWM effect in SOAs has been shown to be a promising method for wavelength conversion. It is attractive since it is independent of modulation format, capable of dispersion compensation and ultra fast. So, wavelength conversion based on FWM effect offers strict transparency, including modulation-format and bit-rate transparency, and is capable of multi-wavelength conversions. However, it has low conversion efficiency and needs careful control of the pola- rization of the input lights [7]. The main drawbacks of wavelength conversion based on FWM are polarization sensitivity and the frequency-shift dependent conversion efficiency.

Wavelength conversion based on XPolM is another promising approach. It uses the optically induced birefringence and dichroism in an SOA and has great potential to offer wavelength conversion with high extinction ratio.

The influence of the nonlinear polarization rotation and the intrinsic and extrinsic SOA parameters on the performance of a wavelength converter based on XGM effect will be the subject of the next section.

\section{WAVELENGTH CONVERTER BASED ON XGM EFFECT IN A SOA STRUCTURE}

Gain saturation of the SOA structure induces nonlinear polarization rotation that can be used to realize wavelength converters [8]. Depending on the system configuration, inverted and non-inverted polarity output can be achieved. Recently, a remarkable wavelength conversion at $40 \mathrm{~Gb} / \mathrm{s}$ with multi-casting functionality based on nonlinear polarization rotation has been demonstrated [18].

In order to evaluate the impact of polarization rotation on the performance of wavelength conversion based on XGM at $40 \mathrm{Gbit} / \mathrm{s}$, we present in this section the concept of this nonlinear effect and the SOA configuration used as wavelength converter whose simulation results will be discussed in the next section.

\section{CROSS-POLARIZATION MODULATION EFFECT}

The XPolM effect denotes the polarization rotation of a beam propagating in a SOA affected by the polarization and the power of a relatively strong control beam introduced simultaneously into the amplifier. When two signals are injected in the SOA, an additional birefringence and gain compression affects the SOA. The two signals affect one another by producing different phase and gain compression on TE and TM components (because the gain saturation of the TE and TM modes is different). This results in a rotation of the polarization state for each signal. The SOA bias current, and the input signal power are among the parameters that determine the magnitude of the polarization rotation. As a result, the XPolM effect in SOA is then directly related to the TE/TM mode discrepancy of XPM and XGM.

The nonlinear polarization rotation that occurs in the SOA is demonstrated to perform very interesting functionalities in optical networks. However, it is exploited in optical gating, in wavelength conversion, in regeneration and in all-optical switching configurations that are required for wavelength routing in high-speed optical time-division multiplexing networks.

The polarization rotation in a SOA structure can be analyzed and evaluated by the azimuth $(\psi)$ and the ellipticity $(\chi)$ parameters, which are given by:

$\left\{\begin{array}{l}\psi=\frac{1}{2} \arctan \left(\frac{s_{2}}{s_{1}}\right) \\ \chi=\frac{1}{2} \arcsin \left(s_{3}\right)\end{array}\right.$ 
Where: $s_{i}, i \in\{1,2,3\}$ are the normalized Stokes parameters, which can be measured using a polarization analyzer.

\section{PROPOSED WAVELENGTH CONVERSION SCHEME}

In the simulation of the proposed wavelength converter based on XGM effect in a wideband traveling wave SOA (TW-SOA) at $40 \mathrm{Gbit} / \mathrm{s}$, which is presented in Fig. (1), we used available OptiSystem software.

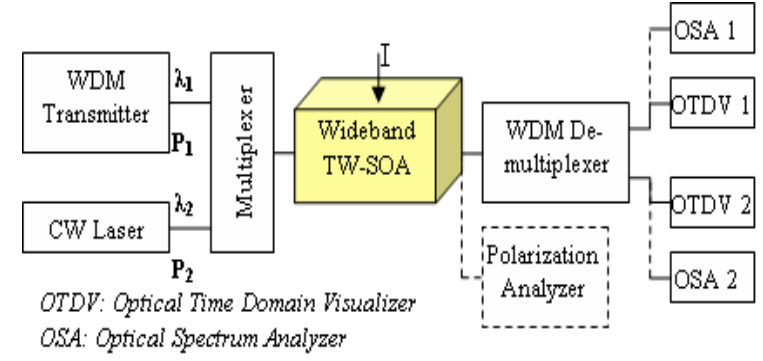

Fig. (1). Schematic of the wavelength converter configuration.

The physical features of the InP/GaInAsP SOA structure are listed in Table 1. While, the pump power $\mathrm{P}_{1}=10 \mathrm{dBm}$ at wavelength $\lambda_{1}=1554 \mathrm{~nm}$ with a pseudorandom bit sequence of length: PRBS $=2^{31}-1$ and the probe power $\mathrm{P}_{2}$ ranges from -40 to $20 \mathrm{dBm}$ at wavelength $\lambda_{2}=1550 \mathrm{~nm}$.

Table 1. SOA Parameters Used in Simulation

\begin{tabular}{|c|c|c|}
\hline Symbol & Description & Value \\
\hline I & Bias current & $200 \mathrm{~mA}$ \\
\hline$\eta_{\text {in }}$ & Input coupling loss & $3 \mathrm{~dB}$ \\
\hline$\eta_{\text {out }}$ & Output coupling loss & $3 \mathrm{~dB}$ \\
\hline $\mathrm{R}_{1}$ & Input facet reflectivity & $5 e-005$ \\
\hline $\mathrm{R}_{2}$ & Output facet reflectivity & $5 e-005$ \\
\hline $\mathrm{L}$ & Active layer length & $500 \mu \mathrm{m}$ \\
\hline $\mathrm{W}$ & Active layer width & $2.5 \mu \mathrm{m}$ \\
\hline $\mathrm{d}$ & Active layer height & $0.2 \mu \mathrm{m}$ \\
\hline$\Gamma$ & Optical confinement factor & $30 \%$ \\
\hline A & $\begin{array}{c}\text { Nonradiative recombination } \\
\text { coefficient }\end{array}$ & $3600000001 / \mathrm{s}$ \\
\hline B & $\begin{array}{l}\text { Bimolecular recombination } \\
\text { coefficient }\end{array}$ & $5.6 \mathrm{e}-016 \mathrm{~m}^{\wedge} 3 / \mathrm{s}$ \\
\hline $\mathrm{C}$ & Auger recombination coefficient & $3 \mathrm{e}-041 \mathrm{~m}^{\wedge} 6 / \mathrm{s}$ \\
\hline $\mathrm{v}_{\mathrm{g}}$ & Group velocity & $75000000 \mathrm{~m} / \mathrm{s}$ \\
\hline $\mathrm{m}_{\mathrm{e}}$ & Effective mass of electron in the $\mathrm{CB}$ & $4.1 \mathrm{e}-032 \mathrm{Kg}$ \\
\hline $\mathrm{m}_{\mathrm{hh}}$ & $\begin{array}{l}\text { Effective mass of a heavy hole in } \\
\text { the VB }\end{array}$ & $4.19 \mathrm{e}-031 \mathrm{Kg}$ \\
\hline $\mathrm{m}_{\mathrm{hl}}$ & $\begin{array}{l}\text { Effective mass of a light hole in the } \\
\text { VB }\end{array}$ & $5.06 \mathrm{e}-032 \mathrm{Kg}$ \\
\hline $\mathrm{A}_{\mathrm{rad}}$ & $\begin{array}{l}\text { Linear radiative recombination } \\
\text { coefficient }\end{array}$ & $100000001 / \mathrm{s}$ \\
\hline $\mathrm{n}_{\mathrm{r}}$ & Active refractive index & 3.7 \\
\hline
\end{tabular}

\section{SIMULATION RESULTS AND DISCUSSION}

An input signal obtained from a WDM transmitter, called the pump, at the wavelength $\lambda_{1}=1554 \mathrm{~nm}$ and a $\mathrm{CW}$ signal, called the probe light, at the desired output wavelength $\lambda_{2}=1550 \mathrm{~nm}$ are multiplexed and launched co-directionally in the wideband TW-SOA. The pump signal modulates the carrier density and consequently the gain of the SOA. The modulated gain modulates the probe light, so that the output probe light, which is known as the converted signal, will contain the information of the input signal, and achieve wavelength conversion (from $\lambda_{1}$ to $\lambda_{2}$ ).
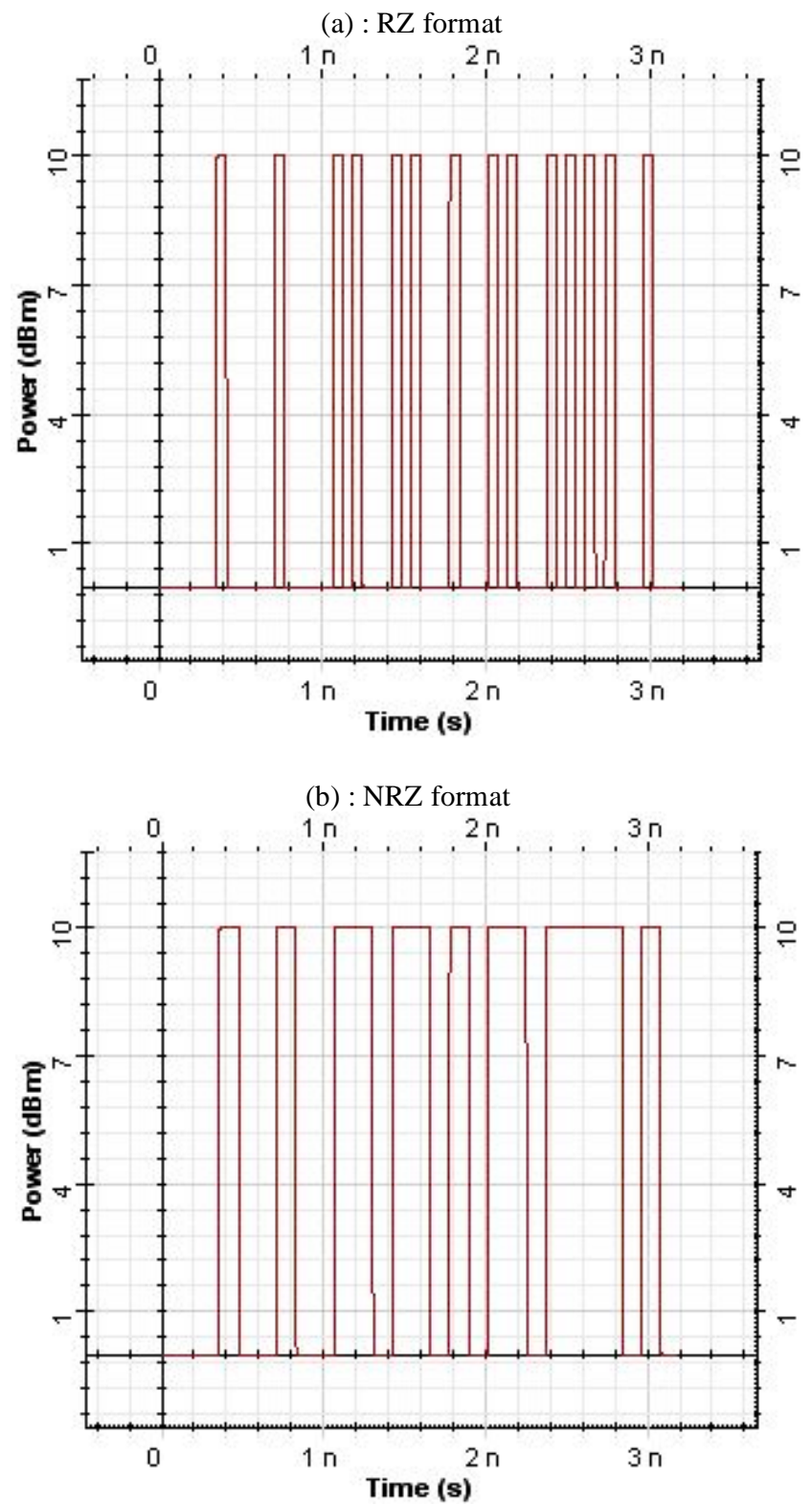

Fig. (2). Original modulated signal at $\lambda_{1}$.

The RZ/ NRZ original information modulated at the signal at $\lambda_{1}=1554 \mathrm{~nm}$, presented in Fig. (2), will be converted to the wavelength $\lambda_{2}=1550 \mathrm{~nm}$. Their spectrums at the output, for the case $\mathrm{P}_{2}=10 \mathrm{dBm}$, are illustrated in Fig. (3). 
(a): at OSA 1

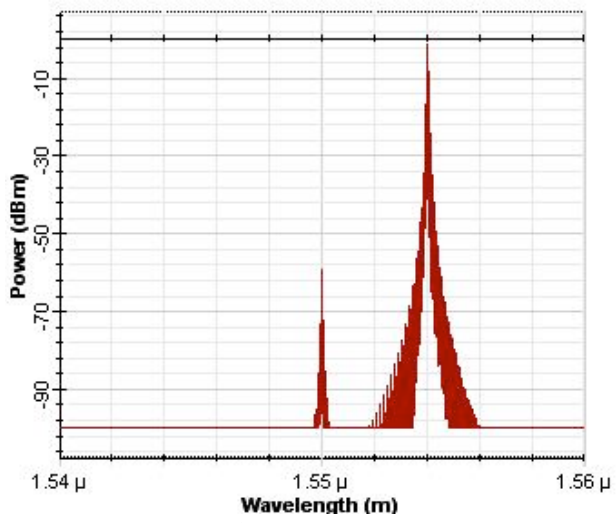

(b): at OSA 2

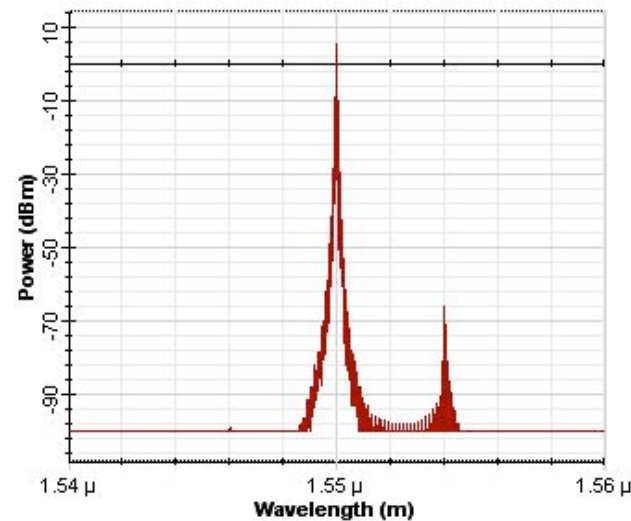

Fig. (3). Spectrum of the output signal power for the case $P_{2}=10$ $\mathrm{dBm}$.

As presented in Fig. (4a), we can notice that the output signal at $\lambda_{1}$ is not inverted. On the other hand, based on

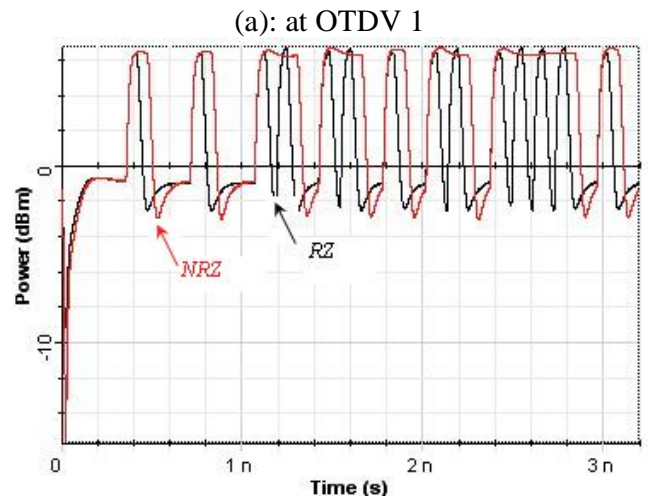

(b): at OTDV 2

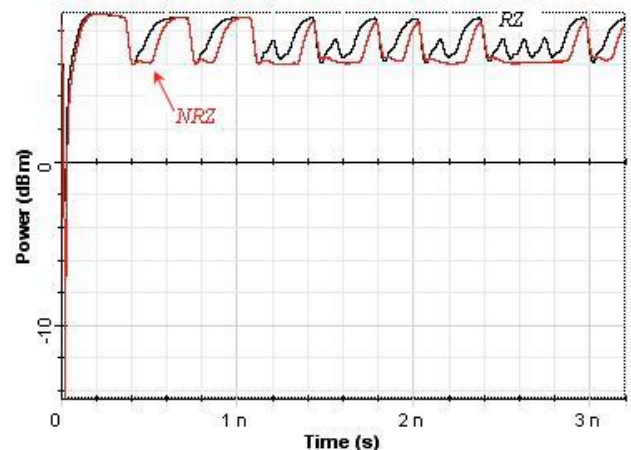

Fig. (4). Evolution of the output signal power by varying the input signal format for the case $\mathrm{P}_{2}=10 \mathrm{dBm}$. (a): at $\lambda_{1}$, (b): at $\lambda_{2}$.
XGM effect, the initial CW signal at $\lambda_{2}$ is modulated at the SOA and the output signal at $1550 \mathrm{~nm}$, for different RZ and NRZ format, becomes the signal depicted in Fig. (4b). As shown, there is an inversion of signal in this case with a remarkable distortion of the signal.

We have also focused on the influence of the variation of the optical confinement factor and the input signal format (RZ/ NRZ) on the output signal power, which is shown in Figs. (5) and (6). Consequently, the output signal can undergo a small variation when the optical confinement factor is increased.

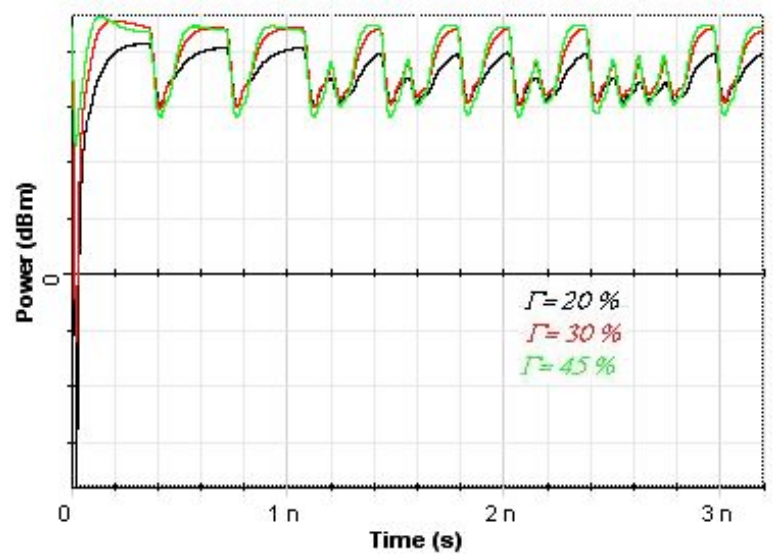

Fig. (5). Output signal power at $\lambda_{2}$ for an RZ signal format and $\mathrm{P}_{2}=10 \mathrm{dBm}$.

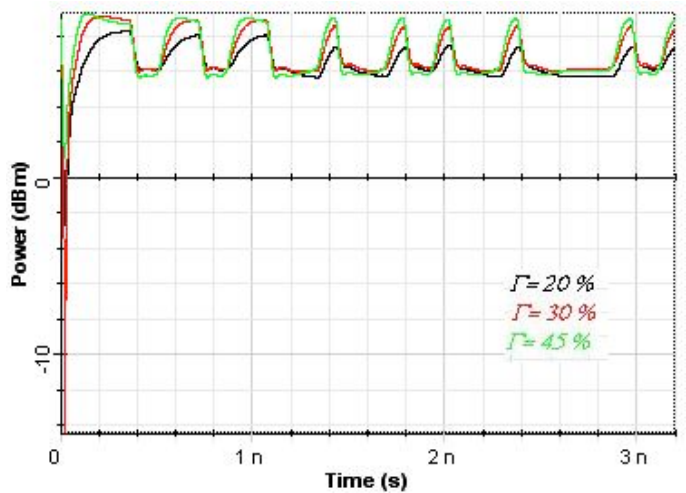

Fig. (6). Output signal power at $\lambda_{2}$ for an NRZ signal format and $\mathrm{P}_{2}=10 \mathrm{dBm}$.

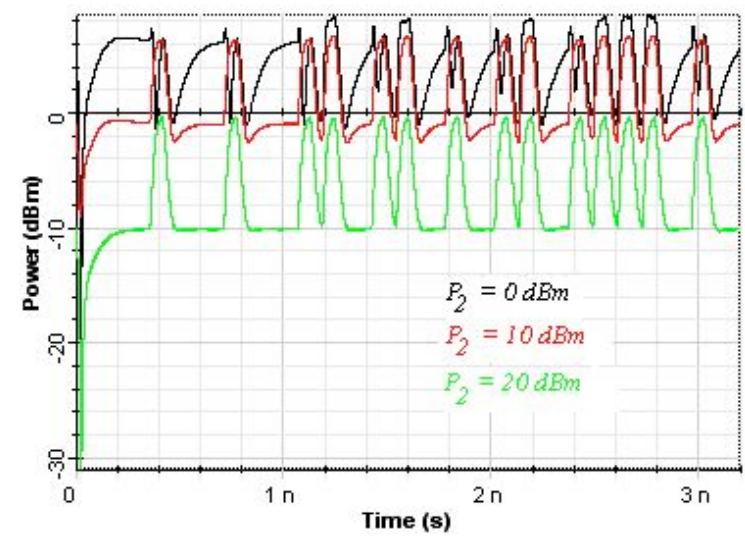

Fig. (7). Output signal by varying the CW input power for an RZ format signal at $\lambda_{1}$, (OTDV 1). 
By varying the $\mathrm{CW}$ input power and the input format signal, we visualized the output signal power by using the OTDV1 and OTDV2, as illustrated in Figs. (7) through (9). So, we can notice that a strong input signal is needed to saturate the SOA gain and thereby to modulate the $\mathrm{CW}$ signal, as it is shown in Figs. (8) and (9b). Also, this is accompanied by a modulation inversion of the output signal which is considered one among the drawbacks of the wavelength conversion using XGM.

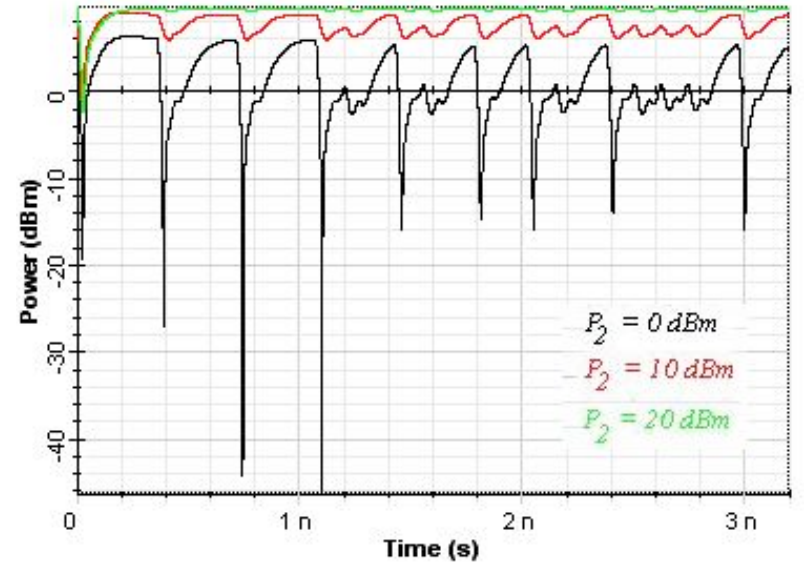

Fig. (8). Output signal by varying the $\mathrm{CW}$ input power for an $\mathrm{RZ}$ format signal at $\lambda_{2}$, (OTDV 2).

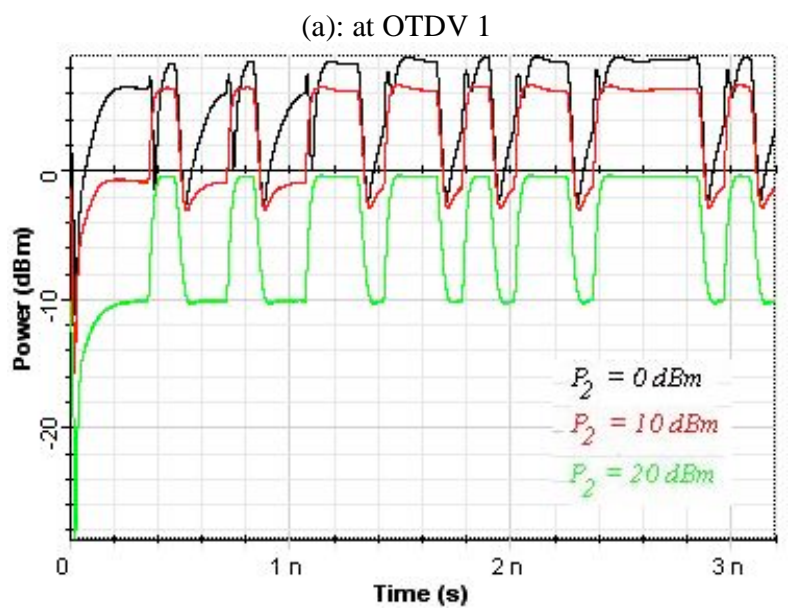

(b): at OTDV 2

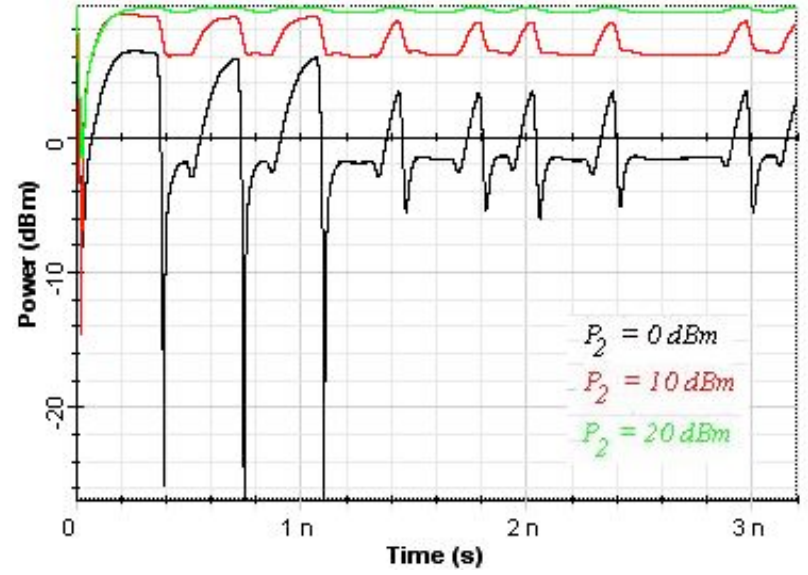

Fig. (9). Evolution of the output signal power as a function of the $\mathrm{CW}$ power for an NRZ format signal.
When the pump is coupled into the structure, owing to the TE/TM asymmetry of the confinement factors, carriers' distributions, the induced nonlinear refractive indices and absorption coefficients of the SOA, there will be an exhibition of birefringent effects induction. Consequently, the linear input polarization will be changed and becomes elliptic at the output as the input power is increased. Thus, the azimuth and ellipticity will be varied at the SOA output, as shown in Fig. (10). A significant change of the polarization state is shown when the $\mathrm{CW}$ input power is high, contrarily for low values that correspond to a linear operating regime. Moreover, this polarization rotation varies not only with the pump power but also as a function of the RZ/NRZ signal format and the optical confinement factor.

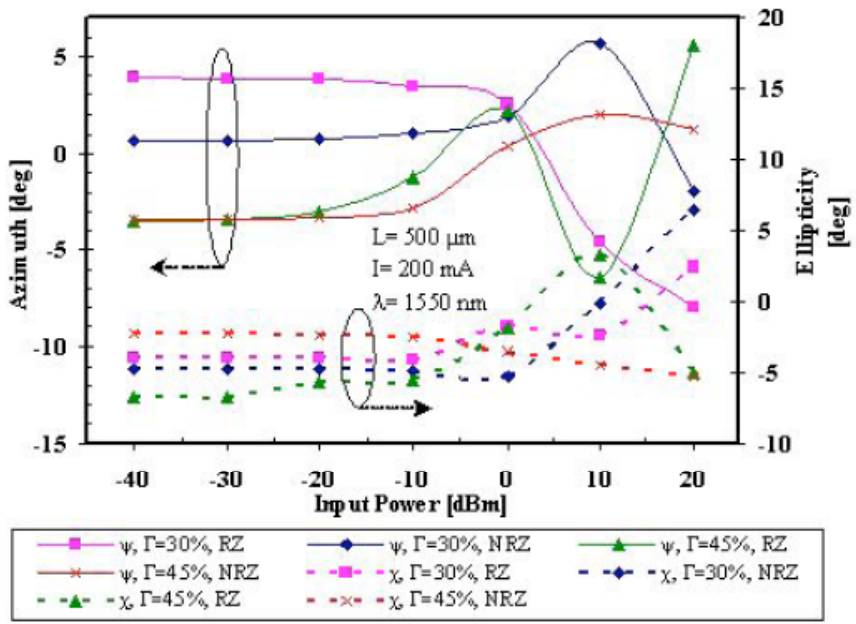

Fig. (10). Curves of the Azimuth and the Ellipticity as function of the input signal power, the signal format and the optical confinement factor.

The amplified spontaneous emission (ASE) noise is considered one of the major processes in analyzing the wavelength converter based on SOAs, because it strongly affects the structure performance. So, the injected signal and the ASE noise interact nonlinearly as they are propagating along the SOA structure. Consequently, the interaction correlates different spectral components of the noise.

A convenient way to treat the impact of noise and characterize its influence on the performance of the wavelength converter is in term of Noise Figure (NF) parameter. It is defined as the ratio between the optical signal to noise ratio (OSNR) of the input signal and the OSNR at the SOA output:

$N F=\frac{(\text { OSNR })_{\text {in }}}{(\text { OSNR })_{\text {out }}}$

The NF represents the amount of degradation in the signal to noise ratio caused by the amplification process.

For low input signal powers, the NF parameter, depicted in Fig. (11), undergoes small variations; and it tends to constant values. In addition, we can note that the NRZ signal format case is more noisy than $\mathrm{RZ}$ one since we have, at $\Gamma=30 \%$ and $\mathrm{P}_{2}=-30 \mathrm{dBm}$ for example, $\mathrm{NF}=9,23 \mathrm{~dB}$ for $\mathrm{RZ}$ format and $\mathrm{NF}=10,12 \mathrm{~dB}$ for $\mathrm{NRZ}$ format. Whereas from certain a threshold point, NF will increased exponentially by augmenting the input power. As well, NF decreases by using SOA having high optical confinement factor. 


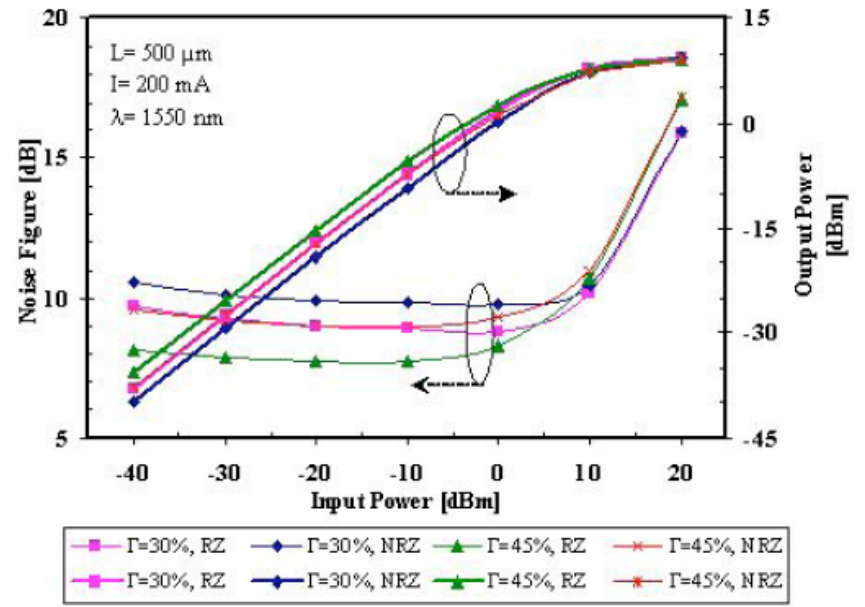

Fig. (11). Evolution of the Noise Figure and the output power as function of the injected signal power for different signal format and various values of the optical confinement factor.

The transfer function, illustrated in Fig. (11), shows that the linear operating regime is exhibited when the input power is low; and then the RZ signal format with high optical confinement factor is the privileged case. The saturation regime will occur as we are increasing the input power; which corresponds to a gain saturation that can cause significant signal distortion at the output of the wavelength converter.

Consequently, in the proposed wavelength converter scheme, we can use a band-pass filter just after the SOA, centered on $\lambda_{2}$ to suppress the spontaneous noise and to extract only the converted signal containing the information of the input signal. Moreover, the discussed wavelength converter configuration can be used to interface access-metro systems with the core network by achieving wavelength conversion of 1310 to $1550 \mathrm{~nm}$ since multi-Gbit/s $1310 \mathrm{~nm}$ transmission technology is used in access and metro networks and the long-haul core network is centered on 1550 nm window.

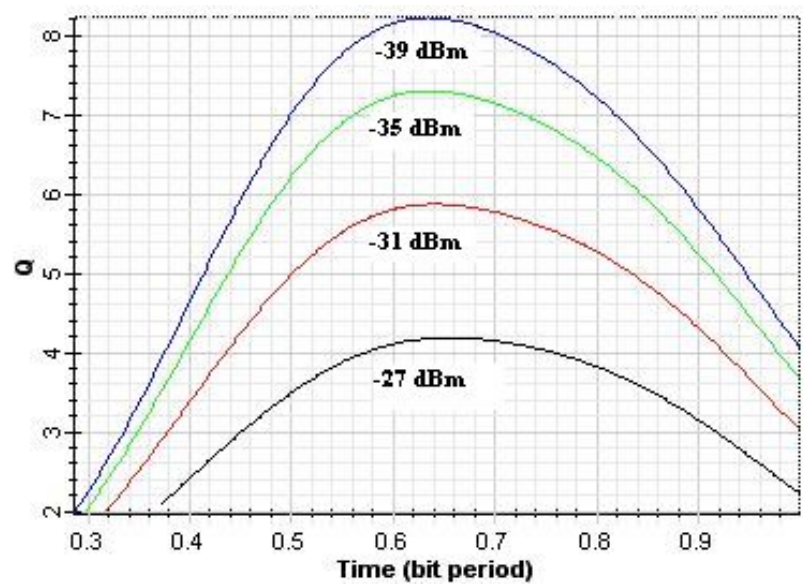

Fig. (12). Curves of the Q-factor for different values of the input power.

In order to deeply analyze the wavelength converter performance, we adopt a wavelength conversion scheme based on an RZ configuration. The used SOA has a bias current $\mathrm{I}=150 \mathrm{~mA}$ and is connected to a receiver composed of a Bessel optical filter centered on $\lambda_{2}$, a photo-detector
PIN, a low pass Bessel filter and a Bit-Error-Rate (BER) analyzer. The default order of the Bessel optical filter was set to 4 in subsequent simulations.

By varying the input power, the maximum value for the Q-factor, the minimum value for the BER, the eye extinction ratio and the eye opening factor versus decision instant are shown below in Figs. (12) to (16).

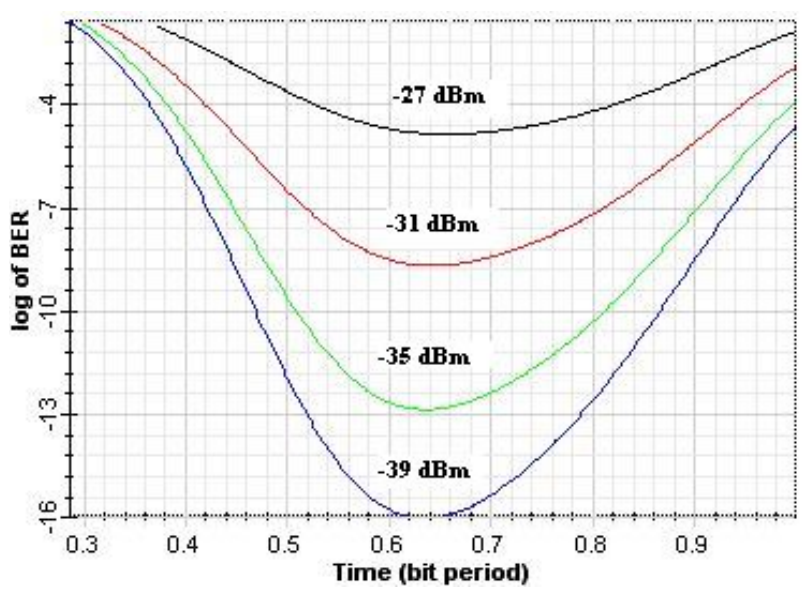

Fig. (13). Evolution of the BER for different values of the input power.

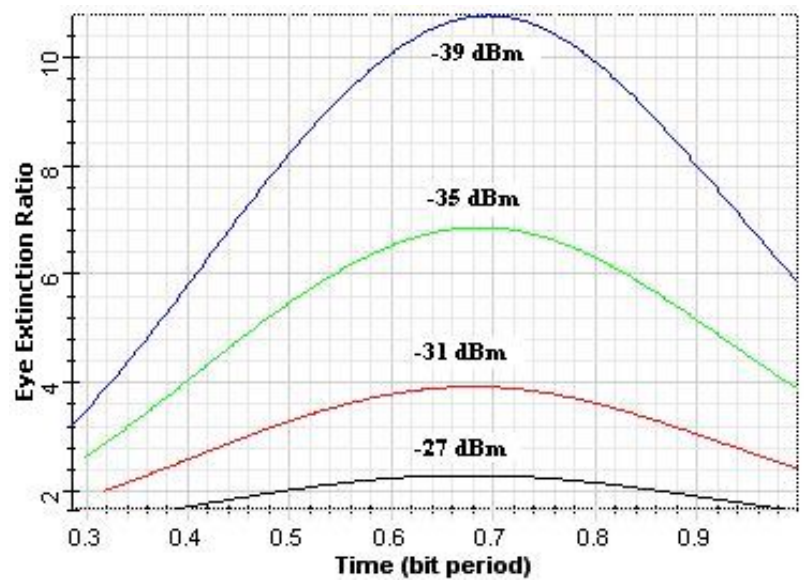

Fig. (14). Evolution of the eye extinction ratio for different values of the input power.

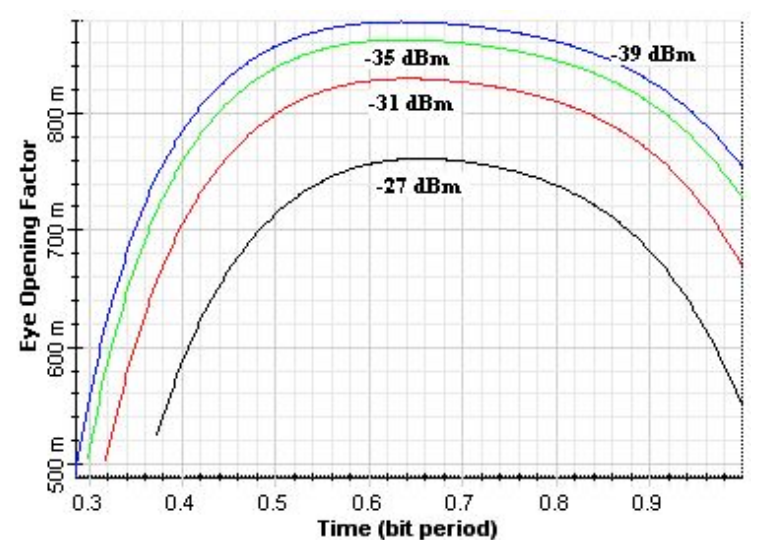

Fig. (15). Evolution of the eye opening factor for different values of the input power. 
The obtained results demonstrate that the optimal point corresponds to an input power equal to $-39 \mathrm{dBm}$. The BER analyzer eye diagram for this case is represented in Fig. (16).

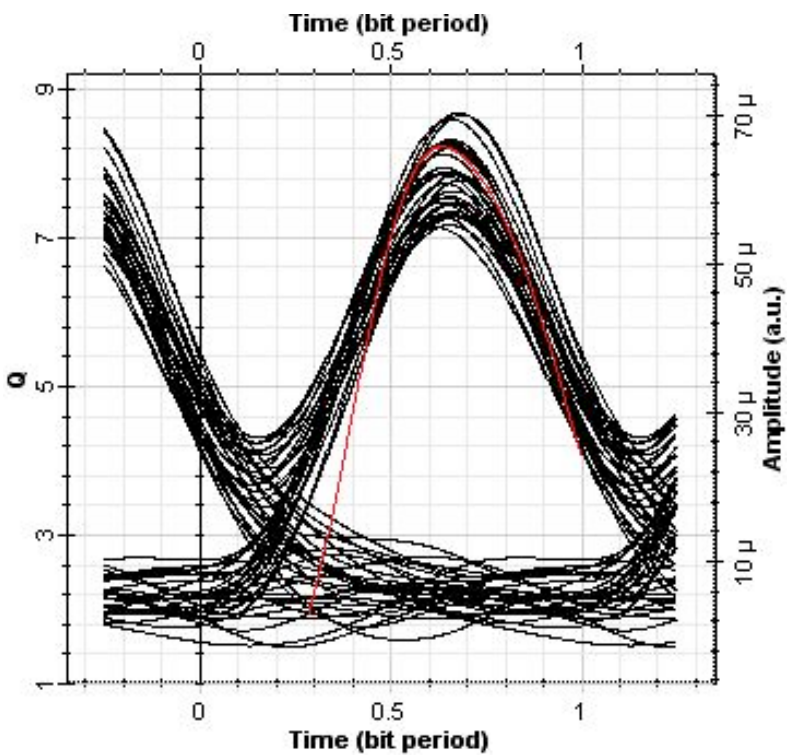

Fig. (16). Evolution the eye diagram and the $Q$ factor for an input power equal to $-39 \mathrm{dBm}$.

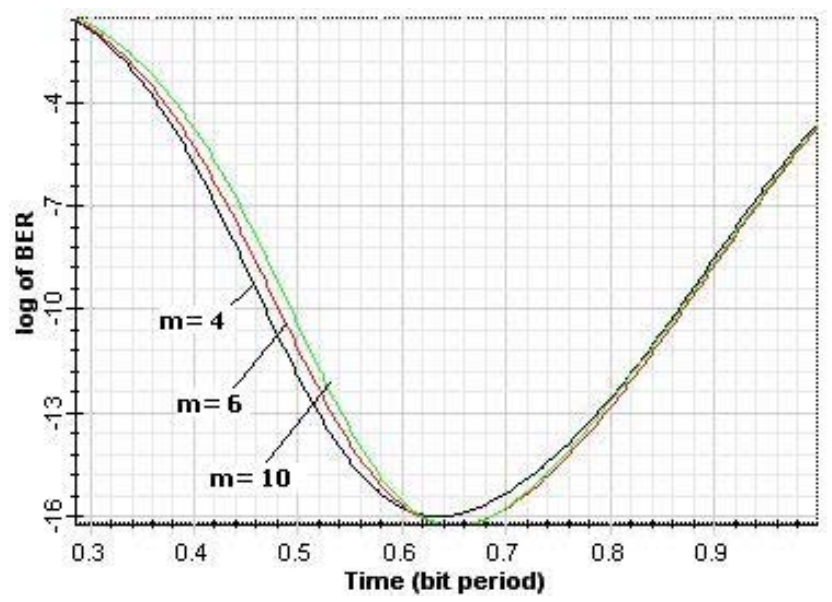

Fig. (17). Evolution of the BER by changing the order of the Bessel low pass filter " $\mathrm{m}$ " for an input power equal to $-39 \mathrm{dBm}$.

As for the order of the Bessel low pass filter at the receiver, it has been also studied to see its effects on performance of the system. It appears from Fig. (17), that the change of the filter order " $\mathrm{m}$ " has a slight variation on the performance of the simulated system.

So, we can conclude that high-speed wavelength conversion seems to be one of the most important functionalities required to assure more flexibility in the next generation optical networks, since wavelength converters, which are the key elements in future WDM networks, can reduce wavelength blocking and offer data regeneration.

\section{CONCLUSION}

We have focused on the impact of nonlinear polarization rotation and the influence of noise on the performance of a wavelength converter based on XGM effect in a SOA structure, which is notable especially when using low input power. Moreover, the case of RZ signal format with high optical confinement factor is privileged in practical operation for assuring better operating conditions of the XGM wavelength converter. As well, performance of the improved wavelength conversion system has been evaluated via the analysis of quality factor and bit error rate.

\section{REFERENCES}

[1] Wei CC, Huang MF, Chen J. Enhancing the frequency response of cross-polarization wavelength conversion. IEEE Photon Technol Lett 2005; 17: 1683-5.

[2] Tzanakaki A, O'Mahony MJ. Analysis of tunable wavelength converters based on cross-gain modulation in semiconductor optical amplifiers operating in the counter propagating mode. Proceedings of IEE Optoelectronics; Feb 2000; Institution of Electrical Engineers, 2000; 147: pp. 49-55.

[3] Ellis AD, Kelly AE, Nesset D, Pitcher D, Moodie DG, Kashyap R. Error free $100 \mathrm{~Gb} / \mathrm{s}$ wavelength conversion using grating assisted cross gain modulation in $2 \mathrm{~mm}$ long semiconductor amplifier. Electron Lett 1998; 34: 1958-9.

Matsumoto A, Nishimura K, Utaka K, Usami M. Operational design on high-speed semiconductor optical amplifier with assist light for application to wavelength converters using cross-phase modulation. IEEE J Quantum Electron 2006; 42: 313-23.

[5] Fu S, Dong J, Shum P, Zhang L, Zhang X, Huang D. Experimental demonstration of both inverted and non-inverted wavelength conversion based on transient cross phase modulation of SOA. J Optics Express 2006; 14: 7587-93.

[6] Contestabile G, Presi M, Ciaramella E. Multiple wavelength conversion for WDM multicasting by FWM in an SOA. IEEE Photon Technol Lett 2004; 16: 1775-7.

[7] Politi C, Klonidis D, O’Mahony MJ. Dynamic behavior of wavelength converters based on FWM in SOAs. IEEE J Quantum Electron 2006; 42: 108-25.

[8] LiuY, Hill MT, Tangdiongga E, et al. Wavelength conversion using nonlinear polarization rotation in a single semiconductor optical amplifier. IEEE Photon Technol Lett 2003; 15: 90-2.

[9] Durhuus T, Mikkelsen B, Joergensen C, Danielsen SL, Stubkjaer KE. All-optical wavelength conversion by semiconductor optical amplifiers. IEEE J Lightwave Technol 1996; 14: 942-54.

[10] Joergensen C, Danielsen SL, Stubkjaer KE, et al. All-optical wavelength conversion at bit rates above $10 \mathrm{~Gb} / \mathrm{s}$ using semiconductor optical amplifiers. IEEE J Select Top Quantum Electron 1997; 3: 1168-80.

[11] Nakamura S, Ueno Y, Tajima K. 168-Gb/s all-optical wavelength conversion with a symmetric Mach-Zehnder-type switch. IEEE Photon Technol Lett 2001; 13: 1091-3.

[12] Manning RJ, Yang X, Webb RP, Giller R, Garcia Gunning FC, Ellis AD. The "turbo-switch" a novel technique to increase the high-speed response of SOAs for wavelength conversion. Proceedings of Optical Fiber Communication Conference and Exposition and The National Fiber Optic Engineers Conference OFC/NFOEC; Mar. 2006; Optical Society of America, Anaheim, USA; paper OWS8; 2006.

[13] Liu Y, Tangdiongga E, Li Z, et al. Error-free 320-Gb/s all-optical wavelength conversion using a single semiconductor optical amplifier. IEEE J Lightwave Technol 2007; 25: 103-8.

[14] Said Y, Rezig H, Bouallegue A. Cross-polarization modulation effects in long semiconductor optical amplifiers. Proceedings of 1 st IEEE International Conference on Transparent Optical Networks Mediterranean Winter Conference ICTON-MW; Dec. 2007; Sousse, Tunisia; 2007.

[15] Said Y, Rezig H, Bouallegue A. On the application of long SOAs in linear operating and saturation regimes: exploitation in high bit rate optical networks. Proceedings of 13th IEEE International Symposium on Computers and Communications ISCC; Jul. 2008; Marrakech, Morocco; 80-3; 2008.

[16] Danielsen S, Hansen P, Stubkjaer K. Wavelength conversion in optical packet switching. IEEE J Lightwave Technol 1998; 16: 2095-108. 
[17] Leuthold J, Joyner CH, Mikkelsen B, et al. 100Gbit/s all-optical wavelength conversion with integrated SOA delayed-interference configuration. Electron Lett 2000; 36: 1129-30.
[18] Contestabile G, Calabretta N, Presi M, Ciaramella E. Single and multicast wavelength conversion at $40 \mathrm{~Gb} / \mathrm{s}$ by means of fast nonlinear polarization switching in an SOA. IEEE Photon Technol Lett 2005; 17: 2652-4.

Received: November 11, 2009

Revised: December 30, 2009

Accepted: January 02,2010

(c) Said et al.; Licensee Bentham Open.

This is an open access article licensed under the terms of the Creative Commons Attribution Non-Commercial License (http://creativecommons.org/licenses/by$\mathrm{nc} / 3.0 /$ ), which permits unrestricted, non-commercial use, distribution and reproduction in any medium, provided the work is properly cited. 